\title{
A importância do pré-natal como momento do diagnóstico da infecção pelo HIV em
}

\section{gestantes}

The importance of Prenatal Care as a time to diagnose HIV infection in pregnant women

La importancia de la atención prenatal como momento del diagnóstico de la infección por VIH en mujeres embarazadas 


\begin{abstract}
Resumo
O objetivo deste estudo foi avaliar a importância da assistência pré-natal na profilaxia da transmissão vertical do HIV (TVHIV). Trata-se de um estudo de coorte retrospectivo no Núcleo Perinatal da Universidade do Estado do Rio de Janeiro. A população de estudo foi constituída por 323 gestantes HIV-positivo e seus bebês, que nasceram na maternidade no período de 2007 a 2018. As gestantes foram separadas de acordo com o momento do diagnóstico da infecção pelo HIV: Grupo 1: diagnóstico durante a gravidez e, Grupo 2: diagnóstico prévio à gravidez. Os dados foram coletados de registros hospitalares das gestantes e dos recém-nascidos. As variáveis utilizadas foram os dados da gestação, parto, puerpério e do acompanhamento do RN. Não houve diferença significativa quanto ao estado conjugal, cor e idade das gestantes. O início do pré-natal após o primeiro trimestre da gestação ocorreu em $64,1 \%$ do grupo 1 e em 43,8\% do grupo 2. Das gestantes que descobriram o diagnóstico na gestação, 82,4\% utilizaram TARV por mais de 4 semanas. Nos casos referentes aos recém-nascidos contaminados $(1,2 \%)$, o número de consultas prénatais foi $<6$ em três dos quatro casos, somente uma das quatro mães utilizou a TARV pelo tempo necessário e a carga viral no terceiro trimestre foi superior a 1.000 cópias $/ \mathrm{mL}$ em três casos e desconhecida em um caso. O pré-natal tem sido um momento significativo para o diagnóstico do HIV e início precoce da profilaxia da TVHIV, com bons resultados perinatais.
\end{abstract}

Palavras-chave: Transmissão vertical de doença infecciosa; Infecções por HIV; Gestação; Cuidado pré-natal.

\begin{abstract}
The objective of this study was to evaluate the importance of prenatal care in the prophylaxis of mother-to-child transmission of HIV (MTCT). This is a retrospective cohort study at the Perinatal Center of the State University of Rio de Janeiro. The study population consisted of 323 HIV-positive pregnant women and their babies, who were born at the maternity hospital from 2007 to 2018. The pregnant women were separated according to the moment of diagnosis of HIV infection: Group 1: diagnosis during pregnancy and, Group 2: diagnosis prior to pregnancy. Data was collected from hospital records of pregnant women and newborns. The variables used were data on pregnancy, delivery, puerperium, and NB follow-up. There was no significant difference regarding marital status, color, and age of the pregnant women. The beginning of prenatal care after the first trimester of pregnancy occurred in $64.1 \%$ of group 1 and in $43.8 \%$ of group 2. Of the pregnant women who discovered the diagnosis during pregnancy, $82.4 \%$ used ART for more than 4 weeks. In the cases of contaminated newborns $(1.2 \%)$, the number of prenatal visits was < 6 in three of the four cases, only one of the four mothers used ART for the necessary time, and the viral load in the third trimester was higher than 1,000 copies/mL, in three cases and, unknown in one case. Prenatal care has been a significant time for HIV diagnosis and early initiation of MTCT, with good perinatal outcomes.
\end{abstract}

Keywords: Vertical infectious vertical transmission; HIV infections; Pregnancy; Prenatal care.

\title{
Resumen
}

El objetivo de este estudio fue evaluar la importancia de la atención prenatal en la profilaxis de la transmisión vertical del VIH (TVVIH). Se trata de un estudio de cohorte retrospectivo en el Centro Perinatal de la Universidad Estatal de Río de Janeiro. La población de estudio estuvo conformada por 323 gestantes VIH positivas y sus bebés, quienes nacieron en la maternidad entre 2007 y 2018. Las gestantes fueron separadas según el momento del diagnóstico de infección por VIH: Grupo 1: diagnóstico durante el embarazo y, Grupo 2: diagnóstico previo al embarazo. Los datos se obtuvieron de los registros hospitalarios de mujeres embarazadas y recién nacidos. Las variables utilizadas fueron datos de embarazo, parto, puerperio y del acompañamiento del RN. No hubo diferencia significativa con respecto al estado civil, color y edad de las embarazadas. El inicio de la atención prenatal después del primer trimestre de embarazo ocurrió en el 64,1\% del grupo 1 y en el 43,8\% del grupo 2. De las gestantes que descubrieron el diagnóstico durante el embarazo, el 82,4\% utilizó TAR por más de 4 años. En los casos referidos a recién nacidos infectados $(1,2 \%)$, el número de consultas prenatales fue $<6$ en tres de los cuatro casos, solo una de las cuatro madres utilizó TAR durante el tiempo necesario y la carga viral en el tercer trimestre fue superior a 1.000 copias / mL en tres casos y desconocido en un caso. La atención prenatal ha sido un momento significativo para el diagnóstico del VIH y el inicio precoz de la profilaxis para el TV-VIH, con buenos resultados perinatales.

Palabras clave: Transmissión vertical de enfermedad infecciosa; Infecciones por VIH; Embarazo; Atención prenatal.

\section{Introdução}

A infecção pelo vírus da imunodeficiência humana (HIV) se mostrou um grande desafio para saúde pública no século XX. De acordo com a UNAIDS (2020) havia 38 milhões de pessoas no mundo vivendo com HIV até o fím de 2019, desses, apenas $81 \%$ conheciam seu estado sorológico positivo No Brasil, no período de 2000 até junho de 2020, foram notificadas 134.328 gestantes infectadas com HIV, sendo a faixa etária entre 20 e 24 anos responsável pelo maior número de casos (27,6\%). Em dez anos, houve aumento de 21,7\% na taxa de detecção de HIV em gestantes, o que pode ser explicado, em parte, pela ampliação do diagnóstico no pré-natal e a consequente prevenção da transmissão vertical do HIV (TVHIV). A taxa de 
detecção de Aids em menores de cinco anos tem sido utilizada como monitoramento da TVHIV e observou-se queda de 47,2\% na taxa para o Brasil nos últimos dez anos (Ministério da Saúde, 2020).

De acordo com o Ministério da Saúde (2018), o teste rápido (TR) para diagnóstico do HIV deve ser pedido na primeira consulta pré-natal, que idealmente ocorre no primeiro trimestre da gestação. Se o TR der negativo, realiza-se o aconselhamento pós-teste e o rastreamento é repetido no terceiro trimestre por meio da sorologia anti-HIV. Caso o TR seja positivo, o aconselhamento pós-teste é realizado e a gestante é encaminhada para o seguimento ao pré-natal no serviço de atenção especializada em DST/Aids de referência (Ministério da Saúde, 2012). Grande parte das mulheres portadoras do vírus é identificada no momento da gravidez ou do parto. Observa-se que entre 38-48\% dessas mulheres chegam às maternidades sem realizar sorologias importantes, como para sífilis e para HIV, necessitando da realização de testes rápidos para que as ações preventivas de transmissão vertical sejam executadas (Araújo, Monte \& Haber 2018). O bom atendimento pré-natal objetiva detectar precocemente quaisquer problemas potenciais, tanto na mãe quanto no feto, para buscar evitá-los e/ou encaminhar as mulheres a especialistas ou hospitais apropriados para seguimento. Esse cuidado precoce e regular pode aumentar as chances de ter um bebê saudável (ACOG e AAP, 2017).

A taxa de transmissão vertical do HIV, sem qualquer intervenção, situa-se em torno de $25,5 \%$, e é possível reduzir para níveis entre zero e 2\%, por meio de intervenções preventivas (Rodrigues Vaz, \& Barros , 2013). A TVHIV ocorre em $35 \%$ dos casos durante a gravidez, $65 \%$ no periparto e o risco de transmissão através da amamentação varia de $7 \%$ a $22 \%$ e o risco está relacionado a fatores como alta carga viral materna, não utilização da terapia antirretroviral (TARV) adequadamente na gestação, intercorrências/comorbidades maternas, rotura de membranas ovulares há mais de quatro horas antes do nascimento e a não realização de cesariana quando a CV é maior do que 1.000 cópias $/ \mathrm{mL}$, após 34 semanas de gestação ( Ministério da Saúde, 2019 e Jourdain ,Mary ,Coeur, Ngo-Giang-Huong , Yuthavisuthi \& Limtrakul , 2007)

Frente ao exposto, o objetivo deste estudo foi avaliar a importância da assistência pré-natal na profilaxia da transmissão vertical do HIV.

\section{Metodologia}

Trata-se de um estudo de coorte retrospectivo, de natureza quantitativa. De acordo com Lima (2011) esse tipo de estudo examina a relação entre características relativas à saúde e outras variáveis de interesse, como elas existem em uma determinada população em um determinado momento. O estudo foi desenvolvido no Núcleo Perinatal do Hospital Universitário Pedro Ernesto da Universidade do Estado do Rio de Janeiro (NP-HUPE/UERJ), situado na zona norte do município do Rio de Janeiro, referência para atendimento especializado ao alto risco materno-fetal. O ambulatório pré-natal recebe mulheres soropositivas que já realizam acompanhamento no próprio hospital ou são referidas pelos sistemas de regulação. As consultas ocorrem nos ambulatórios de Obstetrícia e de Doenças Infecciosas e Parasitárias (DIP). As mulheres HIV-positivo internadas em trabalho de parto podem ou não ter realizado o pré-natal na unidade. Após o parto, os recémnascidos são acompanhados em ambulatório especializado para crianças verticalmente expostas na Pediatria.

A população de estudo foi constituída por 323 gestantes HIV-positivo e seus bebês, que nasceram na maternidade no período de 2007 a 2018. As gestantes foram separadas de acordo com o momento do diagnóstico da infecção pelo HIV: Grupo 1: diagnóstico durante a gravidez e, Grupo 2: diagnóstico prévio à gravidez. Foram excluídas as portadoras de HIV cujo desfecho da gestação foi abortamento, óbito fetal intrauterino ou natimorto.

Os dados foram coletados dos prontuários das gestantes e dos recém-nascidos, em documentos de registros hospitalares e no banco de dados da Secretaria Estadual de Saúde. Em média são 30 mulheres atendidas por ano. As variáveis utilizadas foram os dados da gestação, parto, puerpério e do acompanhamento do RN. 
As variáveis foram definidas como: 1) Variável resposta: ocorrência de TVHIV; 2) Variáveis explicativas: dados sociodemográficos (idade, etnia, escolaridade, estado conjugal e ocupação), dados relacionados à gestação ( momento do diagnóstico da infecção pelo HIV, início e tempo de uso da TARV na gestação, CV no terceiro trimestre, , número de consultas pré-natais), dados relacionados ao parto (via de parto, integridade das membranas, uso de zidovudina (AZT) endovenoso durante o parto), dados relacionados ao recém-nascido (Apgar, peso, uso do AZT e nevirapina).

Os bebês foram considerados como não infectados se apresentassem CV $<5000$ cópias $/ \mathrm{mL}$ em duas amostras, com quatro semanas de intervalo entre elas. Para os que receberam TARV após o nascimento, a primeira CV deveria ser testada, no mínimo, duas semanas após o fim dos antirretrovirais. Também foram consideradas não infectadas, as crianças com pesquisa de anticorpos negativa após os 18 meses de vida. Ao longo da realização do estudo, algumas recomendações foram modificadas e revistas pelo Ministério da Saúde (2018).

Os dados foram descritos através de proporções, médias, desvios-padrão e medianas. Dados perdidos (missing) foram excluídos das análises. A taxa de TVHIV foi calculada pela proporção dos bebês infectados pelo HIV entre aqueles nascidos de mães HIV+, expressa com intervalo de confiança de $95 \%$. As características maternas e do RN foram apresentadas como a relação entre o valor total (n) e o encontrado nos bebês HIV+, sendo comparadas entre os bebês HIV+ e HIV-negativo para avaliar a transmissão vertical. As associações entre transmissão vertical do HIV e possíveis fatores de risco foram testadas através do teste do $\chi 2$ para as variáveis categóricas e o teste $t$ de Student para as variáveis contínuas. O nível de significância foi estabelecido com p-valor $<0,05$. O processo de entrada e análise estatística dos dados foi realizado através do Programa Computacional EPI-INFO 3.5.2.

O Projeto foi aprovado pelo Comitê de Ética em Pesquisa do HUPE/UERJ, CAAE nº 10523719.6.0000.5259.

\section{Resultados}

A população de estudo foi constituída por 323 pares de mães HIV+ e seus bebês, após a exclusão de nove gestantes que sofreram abortamento, óbito fetal intrauterino ou tiveram natimortos. A idade das gestantes variou entre 14 e 44 anos com média de 27,4 $\pm 6,4$ anos e mediana de 27 anos. As adolescentes representaram 12,7\% (41/323), 72,4\% (234/323) apresentava entre 20 a 34 anos e 14,9\% (48/323) delas tinha 35 anos ou mais.

Dentre as 136 mulheres que tinham o ensino fundamental como escolaridade, 75,7\% (103) receberam o diagnóstico do HIV durante a gestação ( $p<0,001)$. Entre as gestantes que não trabalhavam, 61,3\% (111) também pertenciam ao grupo 1 $(\mathrm{p}=0,003)$. Não houve diferença significativa quanto ao estado conjugal, cor e idade das gestantes (Tabela 1).

Tabela 1. Características sociodemograficas das gestantes HIV positivas atendidas o Núcleo Perinatal/HUPE/UERJ, 20072018, de acordo com o momento do diagnóstico da infecção pelo HIV.

\begin{tabular}{|c|c|c|c|c|c|c|}
\hline \multirow{3}{*}{$\begin{array}{c}\text { Variável de } \\
\text { estudo }\end{array}$} & \multicolumn{6}{|c|}{ Momento do diagnóstico } \\
\hline & & \multicolumn{2}{|c|}{ Durante a gestação } & \multicolumn{2}{|c|}{ Fora da Gestação } & \multirow[b]{2}{*}{$\mathbf{p}$} \\
\hline & & Frequência & $\%$ & Frequência & $\%$ & \\
\hline \multirow{2}{*}{ Escolaridade } & fundamental & 103 & 75,7 & 33 & 24,3 & $0,0001 *$ \\
\hline & médio/superior & 76 & 56,7 & 58 & 43,3 & \\
\hline \multirow{2}{*}{$\begin{array}{l}\text { Estado } \\
\text { conjugal }\end{array}$} & unida & 89 & 48,6 & 44 & 48,9 & 0,97 \\
\hline & não-unida & 94 & 51,4 & 46 & 51,1 & \\
\hline \multirow{2}{*}{ Cor } & branca & 66 & 35,9 & 37 & 41,1 & 0,40 \\
\hline & parda/preta & 118 & 64,1 & 53 & 58,9 & \\
\hline \multirow{2}{*}{ Trabalha } & Sim & 70 & 38,7 & 46 & 52,3 & $0,003^{*}$ \\
\hline & Não & 111 & 61,3 & 42 & 47,7 & \\
\hline \multirow{2}{*}{ Idade } & $<20$ anos & 28 & 15,1 & 11 & 12,1 & 0,50 \\
\hline & $\geq 20$ anos & 158 & 84,9 & 80 & 87,9 & \\
\hline
\end{tabular}

Fonte: Autores. 
Em relação ao número de consultas, o grupo 1 realizou menos de 6 consultas pré-natais em 32,6\% (58) e o grupo 2, $18,6 \%$ (16) $(\mathrm{p}=0,01)$. Da mesma forma, o início do pré-natal após o primeiro trimestre da gestação ocorreu em $64,1 \%$ (118) do grupo 1 e em 43,8\% (39) do grupo 2 ( $\mathrm{p}=0,001$ ). (Tabela 2)

Das gestantes que descobriram o diagnóstico na gestação, 82,4\% (98) utilizaram TARV por mais de 4 semanas, enquanto dentre aquelas que já sabiam o diagnóstico antes de engravidar, 95,2\% (59) utilizaram TARV por um período superior a 4 semanas ( $\mathrm{p}=0,01$ ). Não fizeram uso de TARV na gestação, 8,3\% (15) do grupo 1 e 2,2\% (2) do grupo 2 ( $\mathrm{p}=0,04$ ).

Os outros fatores de risco para transmissão vertical do HIV analisados (bolsa rota $>4$ horas, carga viral no $3^{\circ}$ trimestre, peso e Apgar do RN, AZT venoso intraparto, tempo AZT no RN > 4 horas, tipo de parto e uso de nevirapina no RN não mostraram diferenças significativas entre os grupos (Tabela 2).

Tabela 2. Fatores de risco para TVHIV, de gestantes atendidas no Núcleo Perinatal/HUPE/UERJ, 2007-2018, de acordo com o momento do diagnóstico da infecção pelo HIV.

\begin{tabular}{|c|c|c|c|c|c|c|}
\hline \multirow{3}{*}{$\begin{array}{l}\text { Variável de } \\
\text { estudo }\end{array}$} & \multicolumn{6}{|c|}{ Momento do diagnóstico } \\
\hline & & \multicolumn{2}{|c|}{ Durante a gestação } & \multicolumn{2}{|c|}{ Fora da Gestação } & \multirow[b]{2}{*}{$\mathbf{p}$} \\
\hline & & Frequência & $\%$ & Frequência & $\%$ & \\
\hline \multirow{2}{*}{$\begin{array}{l}\text { TARV > } 4 \\
\text { semanas }\end{array}$} & Sim & 98 & 82,4 & 59 & 95,2 & $0,01^{*}$ \\
\hline & Não & 21 & 17,6 & 3 & 4,8 & \\
\hline \multirow{2}{*}{$\mathrm{N}^{\mathrm{o}}$ consultas } & $<6$ & 58 & 32,6 & 16 & 18,6 & $0,01^{*}$ \\
\hline & $\geq 6$ & 120 & 67,4 & 70 & 81,4 & \\
\hline \multirow{3}{*}{$\begin{array}{l}\text { Tempo de bolsa } \\
\text { rota }\end{array}$} & $>4 \mathrm{~h}$ & 34 & 42,5 & 12 & 40,0 & 0,81 \\
\hline & $<4 \mathrm{~h}$ & 46 & 57,5 & 18 & 60,0 & \\
\hline & $<1000$ & 106 & 75,7 & 62 & 84,9 & 0,11 \\
\hline \multirow[t]{2}{*}{ Carga viral $3^{\circ} \mathrm{T}$} & $>1000 \mathrm{ou}$ & & & & & \\
\hline & desconhecida & 34 & 24,3 & 11 & 15,1 & \\
\hline \multirow{2}{*}{ Peso RN } & $<2500 \mathrm{~g}$ & 35 & 19,0 & 11 & 12,1 & 0,15 \\
\hline & $\geq 2500 \mathrm{~g}$ & 149 & 81,0 & 80 & 87,9 & \\
\hline \multirow{2}{*}{ Apgar $5^{\circ} \min$} & $<7$ & 2 & 1,1 & 1 & 1,1 & 0,7 \\
\hline & $\geq 7$ & 179 & 98,9 & 89 & 98,9 & \\
\hline \multirow{2}{*}{$\begin{array}{l}\text { TARV materna } \\
\text { pré-parto }\end{array}$} & Sim & 165 & 91,7 & 89 & 97,8 & $0,04 *$ \\
\hline & Não & 15 & 8,3 & 2 & 2,2 & \\
\hline \multirow{2}{*}{$\begin{array}{l}\text { AZT venoso } \\
\text { intraparto }\end{array}$} & Sim & 157 & 89,7 & 80 & 90,9 & 0,76 \\
\hline & Não & 18 & 10,3 & 8 & 9,1 & \\
\hline \multirow{2}{*}{$\begin{array}{l}\text { Início pré-natal } \\
1^{\circ} \text { Trim }\end{array}$} & Sim & 66 & 35,9 & 50 & 56,2 & $0,001 *$ \\
\hline & Não & 118 & 64,1 & 39 & 43,8 & \\
\hline Tempo AZT & $\leq 4 \mathrm{~h}$ & 135 & 89,4 & 72 & 94,7 & 0,14 \\
\hline após Parto & $>4 \mathrm{~h}$ & 16 & 10,6 & 4 & 5,3 & \\
\hline \multirow{2}{*}{ Tipo de Parto } & Cesáreo & 130 & 69,9 & 71 & 78,0 & 0,15 \\
\hline & Vaginal & 56 & 30,1 & 20 & 22,0 & \\
\hline Uso de & Sim & 12 & 7,5 & 11 & 13,6 & 0,13 \\
\hline Nevirapina & Não & 148 & 92,5 & 70 & 86,4 & \\
\hline
\end{tabular}

Fonte: Autores.

A análise dos fatores relacionados à TVHIV nas mães que tiveram bebês infectados mostrou que as 4 mães cursaram o primeiro grau, o número de consultas pré-natal foi < 6 (3/4 casos) e, em nenhum caso, o tempo de bolsa rota ultrapassou 4 horas. Em relação à TARV, somente uma das quatro mães com bebês infectados utilizou pelo tempo necessário ( $>4$ semanas). A carga viral no terceiro trimestre foi superior a 1.000 cópias $/ \mathrm{mL}$ em três casos e era desconhecida em 1 caso. A tabela 3 mostra a distribuição dos casos de mães HIV-positivo que tiveram bebês infectados. Importante ressaltar que a paciente do caso 1 faleceu de Aids dois anos após o parto. 


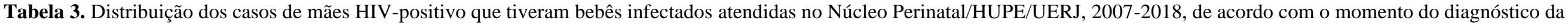
infecção pelo HIV.

\begin{tabular}{|c|c|c|c|c|c|c|c|c|c|c|c|c|c|c|c|c|c|c|c|c|c|}
\hline Casos & Ano & Etnia & $\begin{array}{c}\text { Ida- } \\
\text { de }\end{array}$ & $\begin{array}{c}\text { Estado } \\
\text { conju- } \\
\text { gal }\end{array}$ & Escolaridade & $\begin{array}{l}\text { Ocupa- } \\
\text { ção }\end{array}$ & Gesta & Para & $\begin{array}{l}\text { Abor- } \\
\text { to }\end{array}$ & $\begin{array}{l}\text { Filhos } \\
\text { Vivos }\end{array}$ & $\begin{array}{c}\text { Momento } \\
\text { Diagnós- } \\
\text { tico }\end{array}$ & $\begin{array}{c}\text { Início } \\
\text { do } \\
\text { PN }\end{array}$ & $\begin{array}{l}\mathrm{N}^{o} \text { de } \\
\text { con- } \\
\text { sultas }\end{array}$ & $\begin{array}{c}\text { TARV } \\
\text { antes } \\
\text { do } \\
\text { Parto }\end{array}$ & $\begin{array}{c}\text { Tempo } \\
\text { de uso } \\
\text { TARV } \\
\text { até } \\
\text { parto }\end{array}$ & $\begin{array}{l}\text { Carga } \\
\text { Viral - } \\
3^{\circ} \text { Tri }\end{array}$ & $\begin{array}{c}\text { Tipo } \\
\text { de } \\
\text { Parto }\end{array}$ & $\begin{array}{c}\text { Bolsa } \\
\text { rota } \\
>4 h\end{array}$ & $\begin{array}{c}\text { Peso } \\
\text { do } \\
\text { Bebê }\end{array}$ & $\begin{array}{c}\text { AZT } \\
\text { Oral } \\
\text { no } \\
\text { Bebê }\end{array}$ & $\begin{array}{c}\text { AZTpós- } \\
\text { parto } \\
\text { (tempo) }\end{array}$ \\
\hline $\begin{array}{c}\text { caso } \\
1\end{array}$ & 2015 & Branca & 23 & Unida & $\begin{array}{l}\text { Médio } \\
\text { Incomp. }\end{array}$ & Do Lar & 2 & 1 & 0 & 1 & Gestação & $\begin{array}{c}1^{\circ} \\
\text { Trim. }\end{array}$ & 16 & $S$ & $\begin{array}{c}4 \\
\text { Semanas }\end{array}$ & $>1.000$ & $\mathrm{PC}$ & Não & $3125 \mathrm{~g}$ & S & $3 \mathrm{~h}$ \\
\hline $\begin{array}{c}\text { caso } \\
2\end{array}$ & 2012 & Preta & 31 & $\begin{array}{l}\text { Não } \\
\text { Unida }\end{array}$ & $\begin{array}{l}\text { Funda-mental } \\
\text { Incomp. }\end{array}$ & $\begin{array}{c}\text { Trabalha } \\
\text { Fora }\end{array}$ & 4 & 3 & 0 & 3 & Gestação & $\begin{array}{c}2^{\mathbf{o}} \\
\text { Trim. }\end{array}$ & 5 & $\mathrm{~S}$ & $\begin{array}{c}23 \\
\text { semanas }\end{array}$ & $>1.000$ & $\mathrm{PC}$ & Não & $3275 \mathrm{~g}$ & $\mathrm{~S}$ & $45 \mathrm{~min}$ \\
\hline $\begin{array}{c}\text { caso } \\
3\end{array}$ & 2009 & Parda & 17 & Unida & $\begin{array}{l}\text { Funda-mental } \\
\text { Incomp. }\end{array}$ & Do Lar & 2 & 1 & 0 & 1 & Gestação & $\begin{array}{c}2^{\circ} \\
\text { Trim. }\end{array}$ & 5 & $\mathrm{~N}$ & $\mathrm{~N}$ & $\begin{array}{l}\text { Desco- } \\
\text { nhecida }\end{array}$ & $\mathrm{PV}$ & Não & $2705 \mathrm{~g}$ & S & $50 \mathrm{~min}$ \\
\hline $\begin{array}{c}\text { caso } \\
4\end{array}$ & 2007 & Branca & 26 & Unida & $\begin{array}{c}\text { Funda-mental } \\
\text { Incomp. }\end{array}$ & $\begin{array}{c}\text { Trabalha } \\
\text { fora }\end{array}$ & 5 & 4 & 0 & 2 & Gestação & $\begin{array}{c}3^{\circ} \\
\text { Trim. }\end{array}$ & 2 & $\mathrm{~S}$ & $\begin{array}{c}2 \\
\text { Semanas }\end{array}$ & $>1.000$ & $\mathrm{PC}$ & Não & $2960 \mathrm{~g}$ & $\mathrm{~N}$ & $\mathrm{~N}$ \\
\hline
\end{tabular}

PN: Pré-natal; PC: parto cesáreo; PV: parto vaginal. Fonte: Autores. 


\section{Discussão}

Em relação aos dados sociodemográficos, a mediana de idade das gestantes soropositivas foi de 27 anos. Segundo o Ministério da Saúde (2020), de 1980 a 2016, as gestantes que possuíam entre 25 e 39 anos de idade eram as mais infectadas com HIV no Brasil. Em 2017, entretanto, a faixa etária baixou para 20-24 anos. Os resultados deste estudo mostraram mediana de idade semelhante aos números oficiais. Em relação ao momento do diagnóstico, não houve diferença significativa no momento de diagnóstico da infecção quando estudamos a variável idade.

Quanto à escolaridade, as mulheres que obtiveram o diagnóstico de sorologia positiva para o HIV durante a gestação possuíam menor escolaridade (75,7\% com ensino fundamental) quando comparadas com as mulheres com diagnóstico prévio à gestação. Dessa forma, foram as que apresentaram maior prevalência de bebês infectados. Dentre as mães que transmitiram HIV para seus filhos, todas as quatro mulheres cursaram o ensino fundamental incompleto, e apenas uma chegou ao ensino médio incompleto. Dados semelhantes foram encontrados em estudo que mostrou que há relação inversamente proporcional entre a quantidade de anos de estudo e a ocorrência da infecção pelo HIV em gestantes (Torres \& Luz, 2007).

A ocupação foi outro fator que se mostrou diferença significativa em relação ao momento do diagnóstico: 61,3\% das mulheres que não trabalhavam foram diagnosticadas durante a gestação ( $\mathrm{p}=0,003)$. A TVHIV, entretanto, não foi diferente entre mulheres do lar ou que trabalham fora, assim como não difere nas demais gestantes HIV+, em que não ocorreu TVHIV. Estudo anterior demonstrou resultados semelhantes, em que mais da metade das mulheres HIV+ com ocupação "do lar" estaria associado ao menor nível de escolaridade e, consequentemente, maior dificuldade de obter emprego formal. Em nosso estudo as mulheres com menor escolaridade, foram as que apresentaram maior prevalência de bebes infectados, confirmando estudos anteriores que há relação inversamente proporcional entre a quantidade de anos de estudo e a ocorrência da infecção pelo HIV em gestantes, assim como menor adesão ao pré-natal (Torres \& Luz, 2007).

As outras variáveis sociodemográficas estudadas, estado conjugal e cor, não mostraram relação com o momento do diagnóstico. Em relação ao estado conjugal, estudo anteriores evidenciaram aumento de casos de doenças sexualmente transmissíveis em uniões estáveis. Foi também descrita forte associação entre estar casada/em união estável e soropositividade (Pereira, Costa \& Amaral, 2014). Acreditamos que exista no imaginário social a ideia de quem tem parceiro fixo não se contamina pelo HIV, sendo as medidas preventivas negligenciadas. No nosso estudo constatamos que $75 \%$ das mulheres que transmitiram verticalmente o HIV eram unidas, o que corrobora com os resultados citados anteriormente. Em relação a cor, não houve diferença em relação ao momento do diagnóstico ou à transmissão vertical entre mulheres brancas e pretas/pardas.

O período pré-natal tem se configurado como momento especialmente adequado para o diagnóstico da infecção pelo HIV na mulher, pela universalidade da testagem e pela redução das chances da transmissão vertical para o bebê que sem o adequado planejamento e seguimento feito no pré-natal, é da ordem de $15 \%$ a $45 \%{ }^{13}$. Tem sido também observado no discurso das gestantes que existe deficiência na oferta do teste anti-HIV entre a população feminina fora da gestação, pois a maioria só conhece a oferta da sorologia durante o pré-natal (Silva, Araújo \& Paz ,2008).

A literatura mostra que a redução da transmissão vertical para níveis entre 1 a 2\%, é possível com a aplicação de todas as intervenções preconizadas pela Comissão Nacional de Incorporação de Tecnologias no SUS (Comissão Nacional de Incorporação de tecnologias do SUS, 2020). No entanto, para realizar todas essas intervenções é preciso agir precocemente. Em nosso estudo o início do pré-natal após o primeiro trimestre da gestação ocorreu em 64,1\% (118) do grupo 1 e em 43,8\% (39) do grupo 2 ( $\mathrm{p}=0,001$ ), o que demonstra a necessidade de melhorar a captação precoce das gestantes. Dados semelhantes foram observados em um panorama da assistência pré-natal no contexto da atenção básica de saúde e da Estratégia de Saúde da Família (ESF) no município de Vassouras no interior do Rio de janeiro, onde observou-se dificuldades operacionais ou de contingência, como flexibilização do fluxo de atendimento visando captar precocemente a gestante (Serrazina \& Silva , 2019) 
O Ministério da Saúde (2012) preconiza o mínimo de seis consultas durante o pré-natal. No nosso estudo a maioria das mulheres realizou mais de 6 consultas, sendo $67,4 \%$ das que descobriram a infecção por HIV durante o pré-natal e 81,4\% das mulheres com diagnostico prévio $(\mathrm{p}=0,01)$. Tem sido verificado que o maior número de consultas pré-natais contribui para a maior adesão as ações de profilaxia e cumprimento das recomendações dos serviços de saúde, garantindo o cuidado individualizado, holístico, humanizado e resolutivo para essas gestantes (Lima, Costa ,Teles, Damasceno \& Oriá ,2014).

No presente estudo, 82,4\% das mães que receberam o diagnóstico de HIV durante a gestação e 95,2 \% com diagnostico prévio, fizeram uso da TARV por mais de 4 semanas $(\mathrm{p}=0,01)$. O estado de saúde das mulheres grávidas infectadas com o HIV melhorou consideravelmente com a introdução do TARV. Isso resultou na diminuição das taxas de transmissão vertical do HIV (Cooper, Charurat, Mofenson, Hanson \& Pitt,2002 e Kissim, Mandel, Akatova,Belyakov \& Rakhma,2011)

A TARV materna pré-parto foi realizada em $91,7 \%$ das gestantes que descobriram a infecção na gravidez e $97,8 \%$ fora da gravidez $(\mathrm{p}=0,04)$. O AZT venoso intraparto foi feito em $89,7 \%$, diminuindo a taxa da transmissão vertical da maioria das mães. A administração da AZT na gestação e o uso de AZT injetável durante o trabalho de parto, e no recém-nascido, mostraram-se efetivos na redução da taxa de transmissão vertical para $8,3 \%$, segundo estudo multicêntrico realizado nos Estados Unidos e na França (Ministério da Saúde, 2020). Enfatiza-se também que o benefício na acentuada redução da transmissão vertical do HIV com o uso de drogas antirretrovirais ultrapassa os riscos eventuais dos efeitos adversos (Marques, 2006). Assim, a diminuição da transmissão vertical pode ser feita por meio de cobertura antirretroviral efetiva, orientação e cuidado médico adequado, o que reforça a importância do acompanhamento do pré-natal na redução da TVHIV (Redmond \& Mcnamara , 2015).

A maioria dos bebês de mães HIV+ nasceram de cesariana, que é fator protetor para TVHIV quando a carga viral é alta ou desconhecida. Neste estudo não houve diferença entre o tipo de parto nos dois grupos. A cesariana tem sido uma das principais recomendações das diretrizes para prevenir a transmissão vertical quando a CV no $3^{\circ}$ trimestre é > 1.000 ou desconhecida, juntamente com a suspensão da amamentação e a administração de terapia antirretroviral para gestantes e recém-nascidos (Hernando, Alejos, Montero,Perez, Blanco,Giner, Gomez \& Iribarren,2017). Nosso estudo mostrou controle da carga viral $<1.000$ cópias $/ \mathrm{ml}$ do vírus no $3^{\circ}$ trimestre na maioria das gestantes $(75,7 \%$ e $84,9 \%$, respectivamente) e esses fatores podem ter contribuído para a menor transmissão vertical, visto que a ausência de pré-natal e a realização de pré-natal com menos de seis consultas têm sido associados ao diagnóstico tardio de HIV e à coinfecção por sífilis (Acosta, Gonçalves \& Barcellos, 2016)

Em relação à saúde do bebê, este estudo mostrou que 98,9\% dos bebês das mães infectadas apresentaram Apgar no $5^{\circ}$ minuto superior a 7. Outros estudos constataram que o uso da TARV não apresentou associação com baixo escore no Apgar de $5^{\circ}$ minuto. Assim, é possível inferir que o TARV em que pese ser de fundamental importância na prevenção da TVHIV considerando o uso > 4 semanas, parece não se relacionar ao comprometimento imediato do recém-nascido. No nosso estudo, características do recém-nascido como sexo e idade gestacional não se mostraram associadas à infecção pelo HIV, o que também é verificado na literatura (Barral, Oliveira, Lobato, Martinez \& Gonçalves, 2014)

Quando analisamos apenas os casos referentes aos recém-nascidos contaminados, verificamos que todas apresentavam baixa escolaridade, o que corrobora a importância da desigualdade no acesso à informação e, consequentemente, na aquisição de novos conhecimentos e na adesão ao pré-natal, em concordância com o que tem sido observado na literatura (Guimarães, Parente, Guimarães, Ferreira, \& Garnelo, 2018). Também verificamos que o número de consultas pré-natais foi < 6 em três dos quatro casos. Em relação à TARV, somente uma das quatro mães com bebês infectados utilizou pelo tempo necessário (> 4 semanas). A carga viral no terceiro trimestre foi superior a 1.000 cópias $/ \mathrm{mL}$ em três casos e era desconhecida em um caso. No que tange aos procedimentos em conformidade com as recomendações, verificamos que iniciou-se o AZT xarope antes de 4 horas em três dos recém-nascidos e que foram realizadas três cesarianas e um parto vaginal, sendo que em 
nenhum caso o tempo de bolsa rota ultrapassou 4 horas. O tipo de parto tem sido referido como importante fator de risco para TVHIV estando a cesariana associada a menor risco (European Collaborative Study,1992).

Os outros fatores de risco analisados como bolsa rota > 4 horas, peso e Apgar do RN, não administração de AZT venoso intraparto, início da administração de AZT no RN após 4 horas, tipo de parto e uso de nevirapina no RN não foram significativos.

\section{Conclusão}

O pré-natal mostra ser momento importante para o diagnóstico do HIV positivo, principalmente por ser uma das principais oportunidades oferecida as mulheres em idade reprodutiva. O estudo confirmou que é possível a redução das taxas de TVHIV, nas metas propostas pela OMS, quando as recomendações são cumpridas. Entretanto, é necessário melhorar a captação precoce das gestantes no Pré-natal para oferecimento de todas as estratégias precocemente para a redução da transmissão materno fetal.

No que diz respeito aos fatores de risco para transmissão vertical do HIV, foi possível inferir: que a TARV é de fundamental importância na prevenção da TVHIV, considerando que o uso $>4$ semanas está relacionado com melhores desfechos para o recém-nascido. Todas as mães dos bebês que apresentaram testes positivos tinham $\mathrm{CV}$ no $3^{\circ}$ trimestre $>1.000$ cópias $/ \mathrm{mL}$ ou desconhecida, mostrando a importante associação da CV com a TVHIV ( $\mathrm{p}=0,003)$.

A TVHIV no Núcleo Perinatal HUPE-UERJ ocorreu em 1,2\% dos casos e desde 2015 não há ocorrência de nenhum outro caso. Apesar dos resultados observados, cabe destacar que ainda há um caminho a ser percorrido no sentido de qualificar a atenção pré-natal, em especial na captação precoce, oferecimento da testagem e início oportuno da profilaxia da transmissão vertical do HIV.

Como reflexão, aponta-se para a possibilidade de futuros estudos acerca do tema, utilizando os dados para monitoramento das ações e subsídios para a melhoria da qualidade da assistência.

\section{Referências}

Acosta, L. M. W., Gonçalves, T. R. \& Barcellos, N. T. (2016). Coinfecção HIV/sífilis na gestação e transmissão vertical do HIV: um estudo a partir de dados da vigilância epidemiológica. Rev Panam Salud Publica. 40(6), 435-42. https://www.scielosp.org/pdf/rpsp/2016.v40n6/435-442/pt

American College of Obstetricians and Gynecologists (ACOG) and the American Academy of Pediatrics (AAP). (2017). Antenatal care. Guidelines for Perinatal Care. 8th Edition. Washington, DC: American Academy of Pediatrics. https:/www.acog.org/clinical-information/physician-faqs//media/3a22e153b67446a6b31fb051e469187c.ashx

Araújo, E. da C., et al (2018). Avaliação do pré-natal quanto à detecção de sífilis e HIV em gestantes atendidas em uma área rural do estado do Pará, Brasil. Revista Pan-Amazônica de Saúde, 9(1), 33-39. https://dx.doi.org/10.5123/s2176-62232018000100005.

Barral, M. F., Oliveira, G. R., Lobato, R. C., Mendoza-Sassi, R. A., Martínez, A. M. \& Gonçalves, C. V. (2014). Risk factors of HIV-1 vertical transmission (VT) and the influence of antiretroviral therapy (ART) in pregnancy outcome. Rev Inst Med Trop São Paulo. 56(2), 133-8. https://doi.org/10.1590/ S003646652014000200008

Comissão Nacional de Incorporação de Tecnologias no SUS (CONITEC). (2020). Protocolo Clínico e Diretrizes Terapêuticas para Manejo da Infecção pelo HIV em Crianças e Adolescentes. Relatório de recomendação. Brasília, DF. http://conitec.gov.br/images/Consultas/Relator ios/2020/PCDT_PTV_HIV_CP_42_2020.pdf

Cooper, E., Charurat, M., Mofenson, L., Hanson, I. C., Pitt, J., Diaz, C., et al.(2002). Estratégias de combinação de anti-retrovirais para o tratamento de mulheres grávidas infectadas com o HIV-1 e prevenção da transmissão perinatal do HIV-1. J Acquir Immune Defic Syndr. 2002 ; 29 : $484-94$.

European Collaborative Study (1992). Risk factors for mother-to-child transmission of HIV-1. Lancet 1992;339:1007-

Guimarães, W. S. G., et al (2018). Acesso e qualidade da atenção pré-natal na Estratégia Saúde da Família: infraestrutura, cuidado e gestão. Cadernos de Saúde Pública, 34(5), e00110417.018 .https://doi.org/10.1590/0102-311x00110417

Hernando, V., Alejos, B., Montero, M., Pérez-Elias, J., Blanco, J. R., Giner, L., Gómez-Sirvent, J. L., Iribarren, J. A., Bernal, E., \& Bolumar, F. (2017). Reproductive history before and after HIV diagnosis.Medicines fevereiro de 2017. 96, e5991.

Jourdain, G., Mary, J. Y., Coeur, S.L., Ngo-Giang-Huong, N., Yuthavisuthi, P., Limtrakul, A., et al. (2007). Risk factors for in utero or intrapartum mother-tochild transmission of human immunodeficiency virus type 1 in Thailand. J Infect Dis. 2007; 196(11):1629-36. 10.1086/522009. 
Kissin, D. M., Mandel, M. G., Akatova, N., Belyakov, N. A., Rakhmanova, A. G., Voronin, E. E., et al. (2011). Tendências de cinco anos em epidemiologia e prevenção da transmissão do HIV de mãe para filho, S. Petersburgo, Rússia: resultados da vigilância perinatal do HIV. BMC Infect Dis. 11: 292.

Lima, A. C. M. A. C. C, Costa, C. C., Teles, L. M. R., Damasceno, A. K. C. \& Oriá, M. O. B. (2014). Avaliação epidemiológica da prevenção da transmissão vertical do HIV. Acta Paul Enferm. 27(4):311-8. https://doi.org/10.1590/1982-0194201400053

Lima, D. V. M. (2011). Colaboração para publicação em enfermagem: desafio para hoje. Online braz. j. nurs. (online); 10(2) abr-ago. 2011 http://www.objnursing.uff.br/index.php/nursing/article/view/3649/pdf_2

Marques, H. H. de S. (2006). Avaliação crítica dos efeitos adversos do tratamento anti-retroviral no feto, recém-nascido e lactente. Rev. Bras. Ginecol. Obstet. 28(7), 424-430.. http://www.scielo.br/scielo.php?script=sci_arttext\&pid=S0100-72032006000700008\&lng=en\&nrm=iso.

Ministério da Saúde (2018). Protocolo Clínico e Diretrizes Terapêuticas para Manejo da Infecção pelo HIV em Crianças e Adolescentes / Ministério da Saúde, Secretaria de Vigilância em Saúde, Departamento de Vigilância, Prevenção e Controle das Infecções Sexualmente Transmissíveis, do HIV/Aids e das Hepatites Virais. - Brasília :Ministério da Saúde. http://www.aids.gov.br/pt-br/pub/2017/protocolo-clinico-e-diretrizes-terapeuticas-para-manejo-da-infeccaopelo-hiv-em-criancas-e

Ministério da Saúde (2012). Atenção ao pré-natal de baixo risco. Secretaria de Atenção à Saúde. Departamento de Atenção Básica. http://bvsms.saude.gov.br/bvs/publicacoes/cadernos_atencao_basica_32_prenatal.pdf

Ministério da Saúde. (2018). Manual Técnico para Diagnóstico da infecção pelo HIV em adultos e crianças. Secretaria de Vigilância em Saúde, Departamento de Doenças de Condições Crônicas e Infecçoes Sexualmente Transmissíveis. http://www.aids.gov.br/pt-br/node/57787

Ministério da Saúde. (2020). Boletim Epidemiológico HIV/AIDS 2020. Secretaria de Vigilância em Saúde, Departamento de Doenças de Condições Crônicas e Infecçoes Sexualmente Transmissíveis. http://www.aids.gov.br/pt-br/pub/2020/boletim-epidemiologico-hivaids-2020

Ministério da Saúde (2019). Protocolo Clínico e Diretrizes Terapêuticas para Prevenção da Transmissão Vertical de HIV, Sífilis e Hepatites Virais. Brasília DF. http://www.aids.gov.br/pt-br/pub/2015/protocolo-clinico-e-diretrizes-terapeuticas-para-prevencao-da-transmissao-vertical-de-hiv

Pereira, B. de S., et al (2014). Fatores associados à infecção pelo HIV/AIDS entre adolescentes e adultos jovens matriculados em Centro de Testagem e Aconselhamento no Estado da Bahia, Brasil. Ciência \& Saúde Coletiva, 19(3), 747-758. https://doi.org/10.1590/1413-81232014193.16042013

Redmond, A. M., \& McNamara, J. F. (2015). O caminho para eliminação da transmissão vertical do HIV. Jornal de Pediatria, 91(6), 509511. https://doi.org/10.1016/j.jped.2015.08.004

Rodrigues, S. T. C., Vaz, M. J. R., \& Barros, S. M. O. (2013). Transmissão vertical do HIV em população atendida no serviço de referência. Acta Paulista de Enfermagem, 26(2), 158-164. https://doi.org/10.1590/S0103-21002013000200009

Serrazina, M. F., \& da Silva, G. S. V. (2019). Captação da Gestante para Pré-natal precoce. Pró Univer SUS Jan./Jun.; 10 (1): 29- 34 https://doi.org/10.21727/rpu.v10i1.1621

Silva, R. M. de O., Araújo, C. L. F., \& Paz, F. M. T. (2008). A realização do teste anti-hiv no pré-natal: os significados para a gestante. Escola Anna Nery, 12(4), 630-636. https://doi.org/10.1590/S1414-81452008000400004

Torres, S. R., \& Luz, A. M. (2007).Gestante HIV+ e crianças expostas: estudo epidemiológico da notificação compulsória. Rev Gaucha Enferm. 2007;28(4):505-11

UNAIDS (2020) Global AIDS Update : 2020 Tackling entrenched inequalities to end epidemics Estatísticas. https://www.unaids.org/sites/default/files/media_asset/2020_global-aids-report_en.pdf 